\title{
Two-electron ionization in strong laser fields below intensity threshold: signatures of attosecond timing in correlated spectra
}

\author{
Denys I. Bondar* \\ Department of Physics and Astronomy, \\ University of Waterloo, 200 University Avenue West, \\ Waterloo, Ontario N2L 3G1, Canada and \\ National Research Council, 100 Sussex Drive, \\ Ottawa, Ontario K1A 0R6, Canada \\ Wing-Ki $\mathrm{Liu}^{\dagger}$ \\ Department of Physics and Astronomy, \\ University of Waterloo, 200 University Avenue West, \\ Waterloo, Ontario N2L 3G1, Canada \\ Misha Yu. Ivanov $\ddagger$ \\ National Research Council, 100 Sussex Drive, \\ Ottawa, Ontario K1A 0R6, Canada
}

(Dated: October 31, 2018)

\begin{abstract}
We develop an analytical model of correlated two-electron ionization in strong infrared laser fields. The model includes all relevant interactions between the electrons, the laser field, and the ionic core nonperturbatively. We focus on the deeply quantum regime, where the energy of the active electron driven by the laser field is insufficient to collisionally ionize the parent ion, and the assistance of the laser field is required to create a doubly charged ion. In this regime, the electronelectron and the electron-ion interactions leave distinct footprints in the correlated two-electron spectra, recording the mutual dynamics of the escaping electrons.
\end{abstract}

PACS numbers: $32.80 . \mathrm{Fb}, 34.80 . \mathrm{Qb}, 32.80 . \mathrm{Wr}$

*Electronic address: dbondar@sciborg.uwaterloo.ca

$\dagger$ Electronic address: wkliu@sciborg.uwaterloo.ca 
${ }^{\ddagger}$ Electronic address: Misha. Ivanov@nrc-cnrc.gc.ca 


\section{INTRODUCTION}

In strong infrared laser fields, following one-electron ionization of an atom or a molecule, the liberated electron can recollide with the parent ion $[1,2]$. The electron acts as an "atomic antenna" [2], absorbing the energy from the laser field between ionization and recollision and depositing it into the parent ion. Inelastic scattering on the parent ion results in further collisional excitation and/or ionization. Liberation of the second electron during the recollision - the laser-induced e-2e process - is known as correlated, or nonsequential, double ionization (NSDI).

The phenomenon of NSDI was experimentally discovered by Suran and Zapesochny [3] for alkaline-earth atoms (for further experimental investigations of NSDI for alkaline-earth atoms, see, e.g., Refs. [4-6]). In this case, autoionizing double excitations below the second ionization were shown to be extremely important. For a theoretical study of these effects, see, e.g., Ref. [7]. For noble gas atoms, nonsequential double ionization was first observed by L'Huillier et al. (see, e.g., Refs. [8, 9]). The interest to the phenomenon of NSDI grew rapidly after NSDI was rediscovered in 1993-1994 [10, 11], and now for IR fields and higher intensities. Recently, correlated multiple ionization has also been observed [12, 13]. The renewed interest in NSDI has been enhanced by the availability of new experimental techniques that allow one to perform accurate measurements of the angle- and energyresolved spectra of the photoelectrons, in coincidence. Such measurements play a crucial role in elucidating the physical mechanisms behind the NSDI.

From the theoretical perspective, direct $a b$ initio simulations of the photoelectron spectra corresponding to correlated (or nonsequential) double ionization in intense low-frequency laser fields represent a major challenge. Only now such benchmark simulations have become possible [14] for the typical experimental conditions (the helium atom, laser intensity $I \sim$ $10^{15} \mathrm{~W} / \mathrm{cm}^{2}$, laser wavelength $\left.\lambda=800 \mathrm{~nm}\right)$.

What are the physical reasons behind these numerical challenges, which push the modern computational resources to their limit, occupying thousands of processors for weeks at a time? First, they lie in the need to deal with highly nonstationary two-electron dynamics, with electron energies changing by hundreds of $\mathrm{eV}$ on a subfemtosecond time scale, the characteristic amplitudes of electron oscillations reaching several tens of angstroms, and final electron energies ranging from zero to $10^{3} \mathrm{eV}$. Accurate description of such dynamics 
requires attosecond-scale time steps, very large grids, and small grid steps $\sim 0.1 \stackrel{\circ}{A}$.

Second, one needs to analyze the results of such massive calculations, which output a fivedimensional, time-dependent wave function (one spatial dimension is saved by the cylindrical symmetry of the problem in a linearly polarized laser field.) Extracting essential physical processes and mechanisms responsible for correlated double ionization from such massive data arrays is a separate and equally formidable challenge.

In addition to the experimental measurements, especially of the correlated electron spectra $[12,13,15-19]$, tremendous insight into the physics of the problem has been obtained from classical simulations performed in Refs. [20-25]. These papers have demonstrated a variety of the regimes of nonsequential double and triple ionization. Not only do these simulations reproduce key features observed in the experiment, they also give a clear view of the (classical) interplay between the two electrons, the potentials of the laser field, and of the ionic core. They also show how different types of the correlated motion of the two electrons contribute to different parts of the correlated two-electron spectra.

Our goal is to develop a fully quantum, analytical treatment of this problem. It is important that the approach takes into account all relevant interactions - those with the laser field, the ion, and between the electrons - nonperturbatively. Given the complexity of the problem, it is clear that the analytical description will have to incorporate physical understanding of the dynamics gained from the previous experimental and theoretical work.

In particular, the physics of double ionization is different for different intensity regimes, separated by the ratio of the energy of the recolliding electron to the binding (or excitation) energy of the second electron, bound in the ion.

The maximum energy, which the recolliding electron can acquire from the laser field, is $\sim 3.2 U_{p}[1]$, where $U_{p}=F^{2} / 4 \omega^{2}, F$ is the laser field strength, and $\omega$ is the laser frequency (atomic units are used throughout the paper). Even when $3.2 U_{p}$ is far from sufficient to liberate other electrons, experiments have observed correlated ionization [12, 13, 17-19]. As opposed to the more conventional high- $U_{p}$ regime (see, e.g., Refs. [15, 16, 26-35] and references therein), in the low- $U_{p}$ regime the assistance of the laser field during the recollision is crucial.

Here, we focus on this most challenging low- $U_{p}$ regime, where the nonperturbative interplay of all three interactions is crucial. In this regime, existing classical and quantum analysis (see, e.g., Refs. [23, 25, 36]) demonstrates two possibilities of electron ejection after 
the recollision. First, the two electrons can be ejected with little time delay compared to the quarter-cycle of the driving field. Second, the time delay between the ejection of the first and the second electron can approach or exceed the quarter-cycle of the driving field. In these two cases, the electrons appear in different quadrants of the correlated spectrum. If, following the recollision, the electrons are ejected nearly simultaneously, their parallel momenta have equal signs, and both electrons are driven by the laser field in the same direction toward the detector. If, following the recollision, the electrons are ejected with a substantial delay (quarter-cycle or more), they end up going in the opposite directions. Thus, these two types of dynamics leave distinctly different traces in the correlated spectra.

Here, we consider the case in which the two electrons are ejected nearly simultaneously. We show that in this case the correlated spectra should bear clear signatures of the electronelectron and electron-ion interactions after ionization, including the interplay of these interactions. We identify these signatures.

To study NSDI analytically, we use the strong-field eikonal-Volkov approach (SF-EVA) and follow the recipe described in Ref. [37]. Our model complements earlier theoretical work on calculating correlated two-electron spectra using the strong-field S-matrix approach [26]. The key theoretical advance of this work is the ability to include nonperturbatively all relevant interactions for both active electrons: with each other, with the ion, and with the laser field. Electron-electron and electron-ion interactions are included on an equal footing. Our model ignores multiple recollisions and multiple excitations developing over several laser cycles, such as those seen in the classical simulations [22]. This simplification is particularly adequate for the few-cycle laser pulses, as demonstrated in the experiment [38]. According to this experiment, multiple recollisions are noticeably suppressed already for 12 fs pulses at $\lambda=800 \mathrm{~nm}$. For $6-7$ fsec pulses at $\lambda=800 \mathrm{~nm}$, such simplification is justified.

The rest of this paper is organized as follows: In Sec. II, preliminarily comments from the point of view of the Landau-Dykhne adiabatic theory are made. Basing on the SF-EVA, we develop the analytical model of NSDI in Sec. III. The correlated spectra are first calculated within the strong field approximation (SFA) in Sec. IV. In Secs. V and VI, the roles of electron-electron and electron-ion interactions are analyzed. We show how these interactions lead to profound qualitative differences from the SFA-based models in positions and shapes of maxima in the correlated spectra. Finally, the conclusions are made in the final section. 


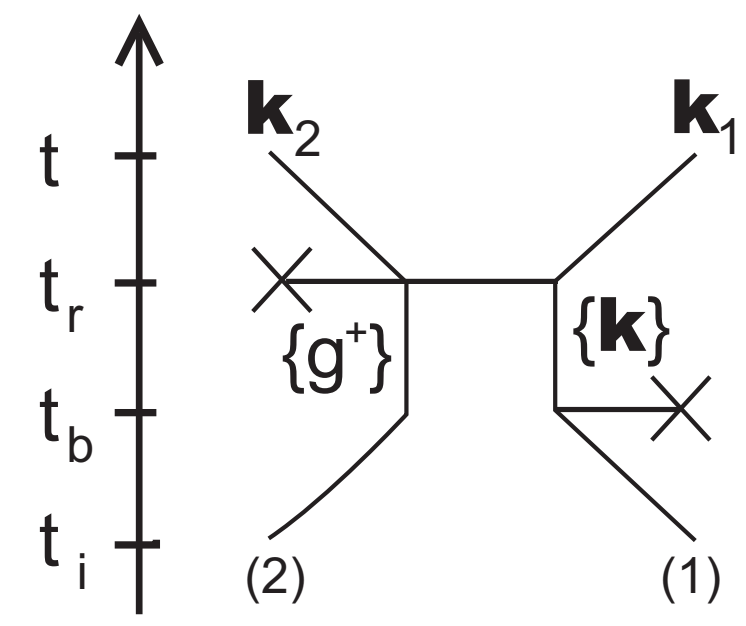

FIG. 1: The diagram of NSDI within the considered regime.

\section{FUNDAMENTAL PRINCIPLES AND BACKGROUND}

The process is shown by the Feynman diagram in Fig. 1. The system begins in the ground state $|g g\rangle$ at time $t_{i}$. At an instant $t_{b}$, intense laser field promotes the first electron to the continuum state $|\mathbf{k}\rangle$; the second electron remains in the ground state of the ion $\left|g^{+}\right\rangle$. Recollison at $t_{r}$ frees both electrons. The symmetric diagram where electrons 1 and 2 change their roles is not shown, but is included in the calculated spectrum.

Despite the fact that the main approach used in this paper is the strong-field eikonal Volkov approximation (SF-EVA), it is methodologically useful to look first at the problem at hand from the point of view of the Landau-Dykhne (LD) adiabatic approximation. Indeed, since the frequency of the laser field is low compared to other relevant energy scales such as the binding energy, the LD approximation is a natural way of tackling the problem. Moreover, the LD approach is an especially handy tool for obtaining results within the strong field approximation, which is used as the zero-order approximation in the SF-EVA [37].

According to the LD method [39, 40] (see also Ref. [41]), if the Hamiltonian of a system $\hat{H}(t)$ is a slowly varying function of time $t$, and $\hat{H}(t) \psi_{n}(t)=E_{n}(t) \psi_{n}(t)(n=i, f)$, then the probability $\Gamma$ of the transition $\psi_{i} \rightarrow \psi_{f}$ is given by (the atomic units are used throughout)

$$
\Gamma \propto \exp \left(-2 \operatorname{Im} \int_{t_{1}}^{t_{0}}\left[E_{f}(t)-E_{i}(t)\right] d t\right)
$$


where $t_{1}$ is any point on the real axis of $t$, and $t_{0}$ is the complex root of the equation

$$
E_{i}\left(t_{0}\right)=E_{f}\left(t_{0}\right)
$$

which lies in the upper half-plane. If there are several roots, we must choose the one that is the closest to the real axis of $t$. It must be stressed that there are no assumptions regarding the form of the Hamiltonian. Further discussions and generalizations of the LD method can be found in Ref. [42-46].

The LD approach has many applications in different areas of physics. In particular, it has been extensively used in strong field physics [41]. For example, the problem of singleelectron ionization can be analyzed within the LD approximation (see Ref. [41, 47-51] and references therein) by setting $E_{i}=-I_{p}$ to be the energy of the ground state ( $I_{p}$ is the ionization potential) and $E_{f}(t)=[\mathbf{k}+\mathbf{A}(t)]^{2} / 2$ to be the energy of the free electron, oscillating in the laser field. Here $\mathbf{k}$ is the final momentum of the electron at the detector, and

$$
\mathbf{A}(t)=-(\mathbf{F} / \omega) \sin (\omega t)
$$

is the vector potential. The results of the LD approach in this form are fully consistent with the usual SFA. Improving the SFA result by incorporating the Coulomb potential is also possible, as we discuss below for the case of two liberated electrons.

Let us apply the general approach Eq. (1) to the two-electron process under consideration. The NSDI has two stages, namely ionization of the first electron and the recollision. Hence, strictly speaking, the LD adiabatic approximation has to be applied to each of the two stages, since the total amplitude of the process is the product of the ionization amplitude and the recollision amplitude. However, it is the second (recollisioin) amplitude that is responsible for the shape of the correlated spectra. The first amplitude only gives the overall height of the spectra, as it determines the overall probability of the recollision. Since at this stage we are only interested in the shape of the correlated spectra, we omit the ionization amplitude from this discussion (it is included later in the full treatment).

As a zero approximation, we define $E_{i}(t)$ and $E_{f}(t)$ for the second part of NSDI without the Coulomb interaction. Before the recollision at the moment $t_{r}$ (Fig. 1), one electron is bound and another is free. The classical energy of the system before $t_{r}$ is

$$
E_{i}(t)=\frac{1}{2}\left[-\mathbf{A}\left(t_{b}(t)\right)+\mathbf{A}(t)\right]^{2}+E_{g^{+}},
$$


where $E_{g^{+}}$denotes the energy level of the second (bound) electron. The time of "birth" (ionization) for the first electron $t_{b}(t)$ is the standard function of the instant of recollision $t_{r}$, which is obtained from the saddle-point S-matrix calculations in the Appendix. In Eq. (3), we have assumed that the recolliding electron has been born at $t_{b}\left(t_{r}\right)$ with zero velocity. After the recollision, both electrons are free and the energy of the system is

$$
E_{f}(t)=\frac{1}{2}\left[\mathbf{k}_{1}+\mathbf{A}(t)\right]^{2}+\frac{1}{2}\left[\mathbf{k}_{2}+\mathbf{A}(t)\right]^{2},
$$

where $\mathbf{k}_{1,2}$ are the asymptotic kinetic momenta at $t \rightarrow \infty$ of the first and second electrons, correspondingly.

Now, substituting Eqs. (3) and (4) into Eq. (1), we obtain the correlated spectrum standard for the strong field approximation (SFA),

$$
\begin{aligned}
& \Gamma_{S F A}\left(\mathbf{k}_{1}, \mathbf{k}_{2}\right) \approx \exp \left(-\frac{2}{\omega} \operatorname{Im} S_{S F A}\left(\mathbf{k}_{1}, \mathbf{k}_{2}\right)\right) \\
& S_{S F A}\left(\mathbf{k}_{1}, \mathbf{k}_{2}\right)=\int_{\operatorname{Re} \varphi_{r}^{0}}^{\varphi_{r}^{0}}\left[\frac{1}{2}\left(\mathbf{k}_{1}+\mathbf{A}(\varphi)\right)^{2}+\frac{1}{2}\left(\mathbf{k}_{2}+\mathbf{A}(\varphi)\right)^{2}-\frac{1}{2}[\mathbf{A}(\varphi)-\mathbf{A}(\Phi(\gamma ; \varphi))]^{2}+I_{p}^{(2)}\right] d \varphi,
\end{aligned}
$$

where the phase of "birth" (ionization) $\Phi(\gamma ; \varphi)$ corresponding to the recollison phase $\varphi$ and the transition point $\varphi_{r}^{0}$ are defined by Eqs. (A11) and (A8) in the Appendix. We will come back to more rigorous analysis of the same spectra in the next section.

The major stumbling block is to account for the electron-electron and the electron-ion interactions on the same footing, nonperturbatively. To include these crucial corrections, we have to include the corresponding Coulomb interactions into $E_{i, f}(t)$. With the nucleus located at the origin, the electron-electron and the electron-core interaction energies are

$$
V_{e e}=1 /\left|\mathbf{r}_{12}(t)\right|, \quad V_{e n}^{(1,2)}=-2 /\left|\mathbf{r}_{1,2}(t)\right|,
$$

correspondingly. Here $\mathbf{r}_{12}(t)=\mathbf{r}_{1}(t)-\mathbf{r}_{2}(t)$ and $\mathbf{r}_{1,2}(t)$ are the trajectories of the two electrons.

However, we immediately see problems. The corrections depend on the specific trajectory, and one needs to somehow decide what this trajectory should be. Note that the classical trajectories $\mathbf{r}_{1,2}(t)$ in the presence of the laser field and the Coulomb field of the nucleus may even be chaotic. The solution to this problem has already been discussed in the original papers by Popov and co-workers [52-55] for single-electron ionization. In the spirit of the eikonal approximation, these trajectories can be taken in the laser field only $[37,54,55]$, so 
that they correspond to the saddle points of the standard SFA analysis. Not surprisingly, in the SFA these trajectories start at the origin,

$$
\mathbf{r}_{1,2}(t)=\int_{t_{0}}^{t}\left[\mathbf{k}_{1,2}+\mathbf{A}(\tau)\right] d \tau
$$

However, here we run into the second problem: the potentials $V_{e e}$ and $V_{e n}^{(1,2)}$ are singular. Consequently, the integral in Eq. (1) is divergent and the result is unphysical. Therefore, such implementation of the Coulomb corrections requires additional care.

The next sections describe a rigorous approach that deals with these two problems, both defining the relevant trajectories and removing the divergences of the integrals.

\section{BASIC FORMALISM}

The key step in dealing with the singularities of the Coulomb potentials during the recollision is to partition the electron-electron and electron-ion interactions in the two-electron Hamiltonian as follows:

$$
\begin{gathered}
V_{e e} \equiv V_{e e}-V_{e e, l n g}+V_{e e, l n g}=V_{e e, l n g}+\Delta V_{e e, s h r}, \\
V_{e n} \equiv V_{e n}-V_{e n, l n g}+V_{e n, l n g}=V_{e n, l n g}+\Delta V_{e n, s h r} .
\end{gathered}
$$

The potential $V_{e e, l n g}$ has a long-range behavior identical to $V_{e e}$, but no singularity at the origin, and $\Delta V_{e e, s h r}$ is singular but short-range potential. The same applies to $V_{e n, l n g}$ and $\Delta V_{e n, s h r}$. We choose

$$
\Delta V_{e n, s h r}(r)=V_{e n}(r) \exp \left(-r / r_{0}\right), \quad \Delta V_{e e, s h r}\left(r_{12}\right)=V_{e e}\left(r_{12}\right) \exp \left[-r_{12} / r_{12}^{(0)}\right]
$$

where $r_{0}$ and $r_{12}^{(0)}$ will be defined later. Note that the partitioning (7) and (8) has been employed originally in the Perelomov-Popov-Terent'ev approach [52-55] for the problem of single-electron ionization.

Now, we can write the Hamiltonian as

$$
\hat{H}(t)=\hat{H}_{s}(t)+\Delta V_{s h r},
$$

where $\Delta V_{s h r} \equiv \Delta V_{e e, s h r}+\Delta V_{e n, s h r}$ and $\hat{H}_{s}$ is the rest, which includes smoothed Coulomb potentials for electron-electron and electron-nuclear interactions, $V_{e e, l n g}$ and $V_{e n, l n g}$. 
To first order in $\Delta V_{s h r}$, the amplitude to find two electrons with momenta $\mathbf{k}_{1}, \mathbf{k}_{2}$ at the detector at the time $t$ is

$$
a\left(\mathbf{k}_{1}, \mathbf{k}_{2}\right)=-i \int_{t_{i}}^{t} d t_{r} \int d^{3} \mathbf{k}\left\langle\mathbf{k}_{1} \mathbf{k}_{2}\left|\hat{U}_{s}\left(t, t_{r}\right) \Delta V_{s h r}\right| g^{+} \mathbf{k}\right\rangle\left\langle\mathbf{k} g^{+}\left|\hat{U}_{s}\left(t_{r}, t_{i}\right)\right| g g\right\rangle .
$$

Approximations in Eq. (9) are first order in $\Delta V_{s h r}$ and the assumption that at the moment of recollision the ion is in its ground state. Both are well justified.

The next step is to approximate the two parts of the evolution: before $t_{r}$ and after $t_{r}$. The key component for correlated spectra is the second part - after $t_{r}$. The main aspect of the first part of the evolution - prior to $t_{r}$ - is to supply an active electron with the required energy.

To simplify the amplitude $\left\langle\mathbf{k}_{1} \mathbf{k}_{2}\left|\hat{U}_{s}\left(t, t_{r}\right) \Delta V_{s h r}\right| g^{+} \mathbf{k}\right\rangle$, we insert the decomposition of unity,

$$
\begin{aligned}
& b\left(\mathbf{k}_{1}, \mathbf{k}_{2}, \mathbf{k}, t_{r}\right)=\left\langle\mathbf{k}_{1} \mathbf{k}_{2}\left|\hat{U}_{s}\left(t, t_{r}\right) \Delta V_{s h r}\right| g^{+} \mathbf{k}\right\rangle= \\
& =\iint d^{3} \mathbf{r}_{1} d^{3} \mathbf{r}_{2}\left\langle\mathbf{k}_{1} \mathbf{k}_{2}\left|\hat{U}_{s}\left(t, t_{r}\right)\right| \mathbf{r}_{1} \mathbf{r}_{2}\right\rangle\left\langle\mathbf{r}_{1} \mathbf{r}_{2}\left|\Delta V_{s h r}\right| g^{+} \mathbf{k}\right\rangle \approx \\
& \approx \iint d^{3} \mathbf{r}_{1} d^{3} \mathbf{r}_{2}\left\langle\mathbf{k}_{1}+\mathbf{A}\left(t_{r}\right), \mathbf{k}_{2}+\mathbf{A}\left(t_{r}\right) \mid \mathbf{r}_{1} \mathbf{r}_{2}\right\rangle\left\langle\mathbf{r}_{1} \mathbf{r}_{2}\left|\Delta V_{s h r}\right| g^{+} \mathbf{k}\right\rangle \times \\
& \exp \left[-i \int_{t_{r}}^{t}\left\{\frac{1}{2}\left[\mathbf{k}_{1}+\mathbf{A}(\tau)\right]^{2}+\frac{1}{2}\left[\mathbf{k}_{2}+\mathbf{A}(\tau)\right]^{2}+\right.\right. \\
& \left.\left.\quad+V_{e e, l n g}\left(\mathbf{r}_{12}(\tau)\right)+V_{e n, l n g}\left(\mathbf{r}_{1}(\tau)\right)+V_{e n, l n g}\left(\mathbf{r}_{2}(\tau)\right)\right\} d \tau\right] .
\end{aligned}
$$

Here we have applied the SF-EVA method [37]. The integral from the nonsingular parts of the electron-electron and electron-ion interactions are calculated along the trajectories in the laser field only. The trajectories

$$
\mathbf{r}_{1,2}(t)=\mathbf{r}_{1,2}+\int_{t_{r}}^{t}\left[\mathbf{k}_{1,2}+\mathbf{A}(\tau)\right] d \tau
$$

and $\mathbf{r}_{12}(t)=\mathbf{r}_{1}(t)-\mathbf{r}_{2}(t)$ begin at the positions $\mathbf{r}_{1}, \mathbf{r}_{2}$ at instant $t_{r}$. The bra-vectors $\left\langle\mathbf{k}_{1,2}+\mathbf{A}\left(t_{r}\right)\right|$ are plane waves. Their distortion by the electron-electron and electron-core interactions appears in the $\left(\mathbf{r}_{1}, \mathbf{r}_{2}\right)$-dependent exponential phase factors in Eq. (10).

Since $\Delta V_{s h r}$ is a short-range potential and $\left|g^{+}\right\rangle$is limited within a characteristic ionic radius, the term $\left\langle\mathbf{r}_{1} \mathbf{r}_{2}\left|\Delta V_{\text {shr }}\right| g^{+} \mathbf{k}\right\rangle$ allows us to fix the initial values of $\mathbf{r}_{1}$ and $\mathbf{r}_{2}$. The characteristic radius for the partitioning of the Coulomb potential into the short-range and long-range parts is set as $r_{0}=r_{12}^{(0)}=1 /\left|E_{g^{+}}\right|$. Therefore, we pull the exponential factor out 
of the integral in Eq. (10) with $r_{0}=r_{12}^{(0)}=1 /\left|E_{g^{+}}\right|$and $r_{1}=r_{2}=0$,

$$
\begin{aligned}
b\left(\mathbf{k}_{1}, \mathbf{k}_{2}, \mathbf{k}, t_{r}\right) \approx\left\langle\mathbf{k}_{1}+\mathbf{A}\left(t_{r}\right) \mathbf{k}_{2}+\mathbf{A}\left(t_{r}\right)\left|\Delta V_{s h r}\right| g^{+} \mathbf{k}\right\rangle \exp \left[-i \int_{t_{r}}^{t}\left\{\frac{1}{2}\left[\mathbf{k}_{1}+\mathbf{A}(\tau)\right]^{2}+\right.\right. \\
\left.\left.+\frac{1}{2}\left[\mathbf{k}_{2}+\mathbf{A}(\tau)\right]^{2}+V_{e e, l n g}\left(\mathbf{r}_{12}(\tau)\right)+V_{e n, l n g}\left(\mathbf{r}_{1}(\tau)\right)+V_{e n, l n g}\left(\mathbf{r}_{2}(\tau)\right)\right\} d \tau\right] .
\end{aligned}
$$

Effects of the long-range tails of $V_{e e}$ and $V_{e n}$ appear in the exponent while the collisional transition is govered by the short-range interaction [37]. The states $|\mathbf{k}\rangle$, so far, represent any convenient basis set of continuum sates in the laser field.

To simplify the amplitude

$$
c\left(\mathbf{k}, t_{r}\right)=\left\langle\mathbf{k} g^{+}\left|\hat{U}_{s}\left(t_{r}, t_{i}\right)\right| g g\right\rangle
$$

we note that the second electron is bound during the whole evolution, and hence we can simplify $c\left(\mathbf{k}, t_{r}\right)$ using single active electron approximation. In this approximation, $\hat{U}_{s}\left(t_{r}, t_{i}\right)$ describes one-electron dynamics in the self-consistent potential of the ionic core,

$$
V_{s c}\left(\mathbf{r}_{1}\right)=\left\langle g^{+}\left|V_{e e, l n g}\left(\mathbf{r}_{12}\right)+V_{e n, l n g}\left(\mathbf{r}_{1}\right)+V_{e n, l n g}\left(\mathbf{r}_{2}\right)\right| g^{+}\right\rangle .
$$

The effective Hamiltonian for evolution between $t_{i}$ and $t_{r}$ is

$$
\hat{H}_{s c}\left(\mathbf{r}_{1}, t\right)=\hat{K}_{1}+V_{s c}\left(\mathbf{r}_{1}\right)+V_{L}\left(\mathbf{r}_{1}, t\right)
$$

where $\hat{K}_{1}$ is the kinetic energy operator and $V_{L}\left(\mathbf{r}_{1}, t\right)$ is the interaction with the laser field. Now the amplitude $c\left(\mathbf{k}, t_{r}\right)$ becomes

$$
c\left(\mathbf{k}, t_{r}\right)=-i \int_{t_{i}}^{t_{r}} d t_{b}\left\langle\mathbf{k}\left|\hat{U}_{s c}\left(t_{r}, t_{b}\right) V_{L}\left(\mathbf{r}_{1}, t_{b}\right)\right| g_{D}\right\rangle \exp \left[i\left|E_{g g}\right|\left(t_{b}-t_{i}\right)\right],
$$

where $\left|g_{D}\right\rangle=\left\langle g_{2}^{+} \mid g g\right\rangle$ is proportional to the Dyson orbital between the ground states of the neutral and ion.

The ionic potential contributes to the propagator in Eq. (13) twice: when the electron leaves the atom near $t_{b}$ and when it returns to the ionic core near $t_{r}$. The contribution "on the way out" introduces standard Coulomb correction [52-57] to the ionization amplitude and hence affects the overall height of the final two-electron distribution. The contribution of $V_{s c}$ "on the way in" affects the spatial structure of the recolliding wave packet. As shown in Refs. [37, 58], for short collision times the Coulomb-laser coupling is small and $V_{s c}$ "on 
the way in" can be included in the adiabatic approximation,

$$
\begin{array}{r}
c\left(\mathbf{k}, t_{r}\right)\left|\mathbf{k}_{e v} g^{+}\right\rangle \approx-i R_{C} \int_{t_{i}}^{t_{r}} d t_{b}\left|\mathbf{k}_{e v} g^{+}\right\rangle\left\langle\mathbf{k}+\mathbf{A}\left(t_{b}\right)-\mathbf{A}\left(t_{r}\right)\left|V_{L}\left(t_{b}\right)\right| g_{D}\right\rangle \times \\
\quad \exp \left[-\frac{i}{2} \int_{t_{b}}^{t_{r}}\left[\mathbf{k}+\mathbf{A}(\tau)-\mathbf{A}\left(t_{r}\right)\right]^{2} d \tau+i\left|E_{g^{+}}\right|\left(t_{r}-t_{b}\right)+i\left|E_{g g}\right|\left(t_{b}-t_{i}\right)\right]
\end{array}
$$

Here $\left|\mathbf{k}_{e v}\right\rangle$ is the field-free continuum wave function in the eikonal approximation, which includes distortions of the incoming plane wave with asymptotic momentum $\mathbf{k}$, $\left\langle\mathbf{k}+\mathbf{A}\left(t_{b}\right)-\mathbf{A}\left(t_{r}\right)\right|$ is a plane wave, and $R_{C}$ is the Coulomb correction to the ionization amplitude which compensates for approximating $\left\langle\mathbf{k}+\mathbf{A}\left(t_{b}\right)-\mathbf{A}\left(t_{r}\right)\right|$ with a plane wave in the matrix element $\left\langle\mathbf{k}+\mathbf{A}\left(t_{b}\right)-\mathbf{A}\left(t_{r}\right)\left|V_{L}\left(t_{b}\right)\right| g_{D}\right\rangle$.

Now, putting together Eq. (14) and Eq. (12) and changing the integration variable $\mathbf{k} \rightarrow \mathbf{k}+\mathbf{A}\left(t_{r}\right)$, we arrive at

$$
\begin{aligned}
& a\left(\mathbf{k}_{1}, \mathbf{k}_{2}\right) \approx-\int_{t_{i}}^{t} d t_{r} \int_{t_{i}}^{t_{r}} d t_{b} \int d^{3} \mathbf{k} \int d^{3} \mathbf{r}_{1} d^{3} \mathbf{r}_{2} \\
& \times \exp \left[-\frac{i}{2} \int_{t_{b}}^{t_{r}}[\mathbf{k}+\mathbf{A}(\tau)]^{2} d \tau+i\left|E_{g^{+}}\right|\left(t_{r}-t_{b}\right)+i\left|E_{g g}\right|\left(t_{b}-t_{i}\right)-\right. \\
& \left.-i \int_{t_{r}}^{t}\left\{\frac{1}{2}\left[\mathbf{k}_{1}+\mathbf{A}(\tau)\right]^{2}+\frac{1}{2}\left[\mathbf{k}_{2}+\mathbf{A}(\tau)\right]^{2}+V_{e e, l n g}\left(\mathbf{r}_{12}(\tau)\right)+V_{e n, l n g}\left(\mathbf{r}_{1}(\tau)\right)+V_{e n, l n g}\left(\mathbf{r}_{2}(\tau)\right)\right\} d \tau\right] \\
& \times\left\langle\mathbf{k}_{1}+\mathbf{A}\left(t_{r}\right), \mathbf{k}_{2}+\mathbf{A}\left(t_{r}\right) \mid \mathbf{r}_{1} \mathbf{r}_{2}\right\rangle\left\langle\mathbf{r}_{1} \mathbf{r}_{2}\left|\Delta V_{\text {shr }}\right| g^{+}, \mathbf{k}_{e v}+\mathbf{A}\left(t_{r}\right)\right\rangle R_{C}\left\langle\mathbf{k}+\mathbf{A}\left(t_{b}\right)\left|V_{L}\left(t_{b}\right)\right| g_{D}\right\rangle .(15)
\end{aligned}
$$

Note that if the Coulomb corrections $V_{e e, l n g}$ and $V_{e n, l n g}$ are ignored in the exponent of Eq. (15), then Eq. (15) coincides with Eq. (A1) within exponential accuracy.

\section{THE CORRELATED TWO-ELECTRON IONIZATION WITHIN THE SFA}

In this section, we find the correlated spectrum of the NSDI by using the strong field approximation (SFA). In the next sections, we will improve the SFA result by employing the SF-EVA [37], i.e., the perturbation theory in action with the SFA result as the zero-order approximation.

Ignoring the Coulomb corrections in Eq. (15) and performing the saddle-point calculations described in the Appendix, we reach the usual SFA expression for the correlated NSDI spectra - Eq. (5).

To illustrate the SFA results, we plot the two-electron correlated spectrum for a system with $I_{p}$ of Ar in Fig. 2. In Fig. 2.A we set $\gamma=0$. Such an SFA spectrum has a peak at 

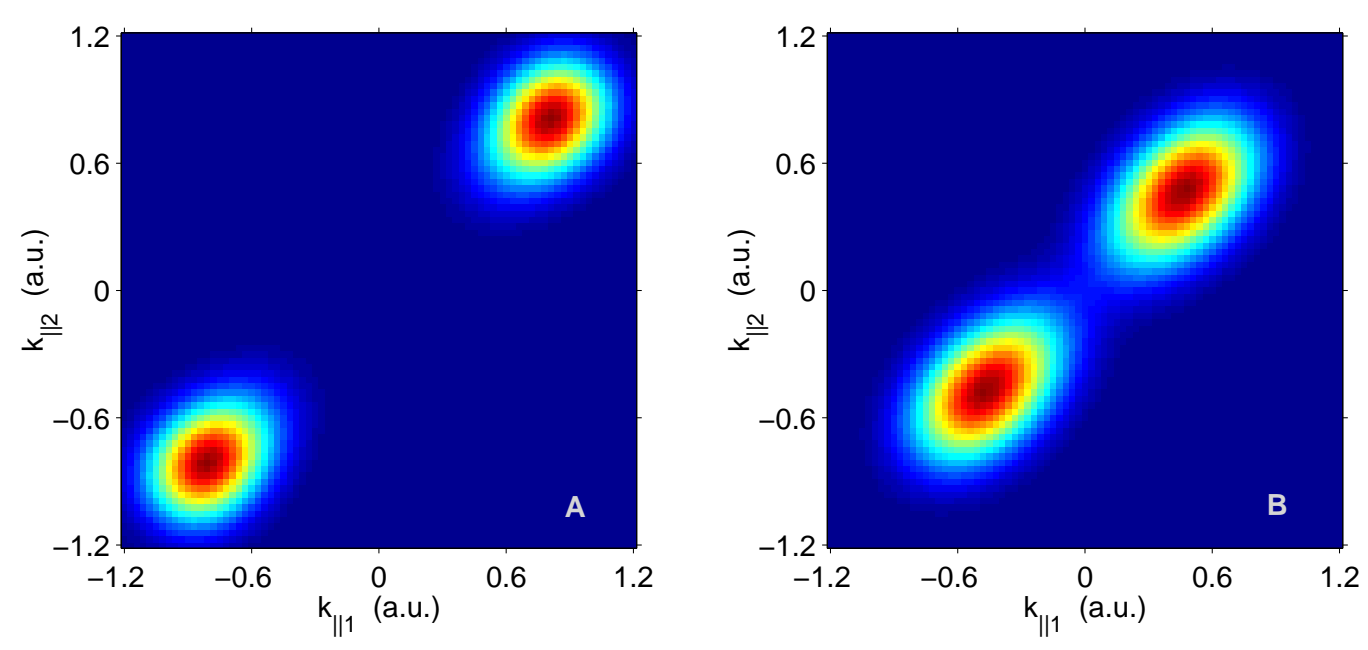

FIG. 2: Correlated two-electron spectra (5) of Ar (linear scale) within the SFA at $7 \times 10^{13} \mathrm{~W} / \mathrm{cm}^{2}$, $800 \mathrm{~nm}\left(k_{\perp 1}=k_{\perp 2}=0\right)$ (a) $\gamma=0$; (b) $\gamma=1.373$. Maxima of figures correspond to probability densities: (a) $1.7 \times 10^{-6}$, (b) $2.9 \times 10^{-15}$.

$k_{\|_{1}}=k_{\|_{2}} \approx-0.78$ a.u., which is the maximum of the vector potential $-F / \omega \approx-0.78$ a.u. The last fact has the following interpretation: NSDI is most efficient when the velocity of the incident electron is maximal. This is achieved near the zero of the laser field, $\mathbf{E}(\varphi)=\mathbf{F} \cos \varphi$, and the maximum of $\mathbf{A}(\varphi)$. An electron liberated at this time could acquire the final drift velocity $\approx-F / \omega$. However, including the correct value of the Keldysh parameter $\gamma$ not only substantially shifts the peak position (Fig. 2.B), but also lowers the maximum by nine orders of magnitude.

\section{ELECTRON-ELECTRON INTERACTION}

In this section, we demonstrate the changes in the correlated spectrum due to the electronelectron repulsion.

Coulomb corrections to the single-electron SFA theory were first introduced by Perelomov, Popov, and Terent'ev $[54,55]$ using the quasiclassical (imaginary time) method (for reviews, see Refs. $[59,60])$. More recently, further improvements to this method have been considered in Refs. [56, 57]. These improvements considered not only subbarrier motion in imaginary time, but also the effects of the Coulomb potential on the phase of the outgoing wave packet in the classically allowed region. These improvements allowed the authors of Refs. [56, 57] to obtain quantitatively accurate results not only for ionization yields, but also for the 
above threshold ionization spectra of direct electrons (i.e., not including recollision). An alternative, but conceptually similar, approach is the SF-EVA [37]. Unlike the two previous methods, the SF-EVA allows a simple treatment of the electron-electron and electron-ion interaction in the two-electron continuum states.

According to the SF-EVA, the contribution of the interaction potentials is calculated along the SFA trajectories,

$$
\mathbf{r}_{1,2}(\varphi)=\frac{1}{\omega} \int_{\varphi_{r}^{0}}^{\varphi}\left[\mathbf{k}_{1,2}+\mathbf{A}(\phi)\right] d \phi
$$

Note that at the moment of recollision $\varphi_{r}^{0}$, the electrons are assumed to be at the origin, $\mathbf{r}_{1,2}\left(\varphi_{r}^{0}\right)=\mathbf{0}$. However, this does not cause any divergence since according to Eq. (15) we have to use the regularized potential $V_{e e, l n g}$.

From Eqs. (7) and (8), the potential energy of electron-electron repulsion along these trajectories is given by

$$
\begin{aligned}
V_{e e, \operatorname{lng}}(\varphi) & =\frac{1}{r_{12}(\varphi)}\left(1-\exp \left[-\frac{r_{12}(\varphi)}{r_{12}^{(0)}}\right]\right), \\
r_{12}(\varphi) & =\left|\mathbf{r}_{1}(\varphi)-\mathbf{r}_{2}(\varphi)\right|=\sqrt{\left[\left(k_{\|_{1}}-k_{\|_{2}}\right) \frac{\varphi-\varphi_{r}^{0}}{\omega}\right]^{2}+\left[\left(k_{\perp 1}-k_{\perp 2}\right) \frac{\varphi-\varphi_{r}^{0}}{\omega}\right]^{2}} .
\end{aligned}
$$

As discused in Sec. III, the parameter $r_{12}^{(0)}$ is set to the ionic radius, $r_{12}^{(0)}=1 / I_{p}^{(2)}$.

The correlated spectrum, which accounts for the electro-electron interaction, has the form

$$
\begin{aligned}
& \Gamma_{e e}\left(\mathbf{k}_{1}, \mathbf{k}_{2}\right) \approx \exp \left(-\frac{2}{\omega} \operatorname{Im}\left[S_{S F A}\left(\mathbf{k}_{1}, \mathbf{k}_{2}\right)+S_{e e}\left(\mathbf{k}_{1}, \mathbf{k}_{2}\right)\right]\right) \\
& S_{e e}\left(\mathbf{k}_{1}, \mathbf{k}_{2}\right)=\int_{\operatorname{Re} \varphi_{r}^{0}}^{\varphi_{r}^{0}} V_{e e, \operatorname{lng}}(\varphi) d \varphi .
\end{aligned}
$$

Figure 3 shows the contribution of electron-electron repulsion to the spectra of NSDI for an atom with $I_{p}$ of $\operatorname{Ar}$ (for experimental data see Ref. [17]).

Comparing Figs. 2 and 3, we readily notice a dramatic influence of electron-electron interaction on the correlated spectra. Electron-electron repulsion splits each SFA peak into two peaks because, due to the Coulomb interaction, two electrons cannot occupy the same volume. Note that the larger the difference between the perpendicular momenta of both of the electrons, the closer is the location of the peaks. 


\section{ELECTRON-ION INTERACTION}

Now we include the electron-ion attraction. The potential energy of electron-ion interaction for the case of two electrons and a single core, after partitioning (7) and (8), is

$$
\begin{aligned}
V_{e n, \operatorname{lng}}(\varphi) & =-2 \sum_{i=1}^{2} \frac{1}{r_{i}(\varphi)}\left(1-\exp \left[-\frac{r_{i}(\varphi)}{r_{0}}\right]\right) \\
r_{1,2}(\varphi) & =\sqrt{\left(k_{\|_{1,2}} \frac{\varphi-\varphi_{r}^{0}}{\omega}+\frac{F}{\omega^{2}}\left(\cos \varphi-\cos \varphi_{r}^{0}\right)\right)^{2}+\left(k_{\perp 1,2} \frac{\varphi-\varphi_{r}^{0}}{\omega}\right)^{2}} .
\end{aligned}
$$

As far as the parameter $r_{0}$ is concerned, we set it equal to $r_{12}^{(0)}=1 / I_{p}^{(2)}$.

Finally, the correlated spectrum of NSDI, which takes into account both electron-electron and electron-ion interactions, reads

$$
\begin{aligned}
\Gamma_{e e+e n}\left(\mathbf{k}_{1}, \mathbf{k}_{2}\right) & \approx \exp \left(-\frac{2}{\omega} \operatorname{Im}\left[S_{S F A}\left(\mathbf{k}_{1}, \mathbf{k}_{2}\right)+S_{e e}\left(\mathbf{k}_{1}, \mathbf{k}_{2}\right)+S_{e n}\left(\mathbf{k}_{1}, \mathbf{k}_{2}\right)\right]\right) \\
S_{e n}\left(\mathbf{k}_{1}, \mathbf{k}_{2}\right) & =\int_{\operatorname{Re} \varphi_{r}^{0}}^{\varphi_{r}^{0}} V_{e n, l n g}(\varphi) d \varphi
\end{aligned}
$$

To illustrate the influence of the electron-ion attraction, we have plotted the correlated spectra of Ar in Fig. 4 for different perpendicular momenta. Comparing Figs. 3 and 4, we conclude that the larger the difference between the perpendicular momenta of the two electrons, the larger is the contribution of the electron-ion interaction. Furthermore, accounting for electron-ion attraction increases the probability of NSDI by 15 orders of magnitude. This occurs because, as in the case of single-electron ionization, electron-core interaction significantly lowers an effective potential barrier. We can also conclude that correlated spectra pictured in Figs. 4.C, 4.D, and 4.E have the biggest contribution to the total probability of NSDI, which is an integral of the probability density over momenta of both of the electrons. Note that, on the one hand, the maximum of the probability density shown in Fig. 4.F is the largest among those presented in Fig. 4, and on the other hand, this maximum is localized in a few pixels; therefore, the integral contribution of Fig. 4.F to the total probability is smaller than Fig. 4.E. Additionally, as one would expect, further increasing of $k_{\perp}$ leads to a decrease of probability density. The correlated spectra in Fig. 4 agree with the experimental data [17] in quadrants one and three. The considered diagram

(Fig. 1) does not contribute to signals in quadrants two and four. Note that taking into account a nonzero value of $\gamma$ is vital to achieve agreement with the experimental data. 
From Eqs. (16) and (18), we can notice that if $r_{12}^{(0)} \rightarrow \infty$ and $r_{0} \rightarrow \infty$, the Coulomb corrections $V_{e e, l n g}$ and $V_{e n, l n g}$ vanish, and the SFA result is recovered. Therefore, we conclude that the radii $r_{12}^{(0)}$ and $r_{0}$ contain the information about the initial position of electrons after they emerged in the continuum. Obviously, the intra-electron distance should be on the order of an ion radius.

\section{CONCLUSIONS}

The analytical quantum-mechanical theory of NSDI within the deeply quantum regime, when the energy of the active electron driven by the laser field is insufficient to collisionally ionize the parent ion, has been formulated based on the SF-EVA approach. On the whole, the presented model agrees with available experimental data [17]. We have defined the quantum-mechanical phase of birth of the active electron (A11), which accurately accounts for tunneling of the recolliding electron in the regime where both the phases $\varphi_{r}$ and $\varphi_{b}$ are complex. Moreover, it has been demonstrated that ignoring such a contribution of tunneling of the active electron fails to agree with the experimental data.

Furthermore, our results show that any attempt to interpret NSDI spectra in this regime in terms of a simple SFA-based streaking model would lead to wrong conclusions on the relative dynamics of the two electrons.

The contributions of the electron-electron and electron-ion interactions have been analyzed. Both play an important and distinct role in forming the shape of the correlated spectra.

The presented model is not able to give the correlated spectra in quadrants two and four. It is because the considered process, when two electrons detach simultaneously from the atom, does not contribute to that area. However, we incline to believe that those parts of the correlated spectra are formed due to recollision and excitation of the ion plus subsequent field ionization [61,62], and it should be noted that this mechanism has been also observed in classical simulations [23]. We are planing to develop an analytical model of such a mechanism in future papers. 


\section{Acknowledgments}

We thank A. Rudenko, O. Smirnova, G.L. Yudin, and M. Spanner for highly stimulating discussions. Financial support to D.I.B. by an NSERC SRO grant is gratefully acknowledged.

\section{APPENDIX A: THE PHASE OF IONIZATION OF THE RECOLLIDING ELEC- TRON AS A FUNCTION OF THE PHASE OF RECOLLISION}

Employing the SFA, we write the formula corresponding to the digram of NSDI (Fig. 1)

$$
\begin{array}{r}
|\Psi(t)\rangle \sim \int_{t_{i}}^{t} d t_{b} \int_{t_{b}}^{t} d t_{r} \int d^{3} \mathbf{k} \hat{U}\left(t, t_{r}\right) \frac{1}{r_{12}}\left|\mathbf{k} g^{+}\right\rangle\left\langle g^{+} \mathbf{k}\left|\hat{V}_{L}\left(t_{b}\right)\right| g g\right\rangle \times \\
\quad \exp \left\{-\frac{i}{2} \int_{t_{b}}^{t_{r}}[\mathbf{k}+\mathbf{A}(\tau)]^{2} d \tau+i\left|E_{g^{+}}\right|\left(t_{r}-t_{b}\right)+i\left|E_{g g}\right|\left(t_{b}-t_{i}\right)\right\},
\end{array}
$$

where $\hat{U}\left(t, t_{r}\right)$ is the evolution operator of the studied system, $r_{12}$ is the distance between

the electrons, $\hat{V}_{L}\left(t_{b}\right)$ is the interaction between the ionized electron and the laser field, and $E_{g^{+}}$and $E_{g g}$ are energies of the states $\left|g^{+}\right\rangle$and $|g g\rangle$, respectively.

We use the saddle point approximation (SPA) to calculate the integrals over $\mathbf{k}$ and $t_{b}$ in Eq. (A1). The phase of the integral over $\mathbf{k}$ has the following form:

$$
S_{1}(\mathbf{k})=-\frac{1}{2} \int_{t_{b}}^{t_{r}}[\mathbf{k}+\mathbf{A}(\tau)]^{2} d \tau
$$

The saddle point of this integral is given by

$$
\mathbf{k}^{*}=\frac{-1}{t_{r}-t_{b}} \int_{t_{b}}^{t_{r}} \mathbf{A}(\tau) d \tau
$$

with the restriction $t_{r} \neq t_{b}$. Note that generally speaking, $\mathbf{k}^{*}$ can be complex since $t_{b}$, as will be clarified below, is complex for $\gamma \neq 0$. The phase of the integral over $t_{b}$ in Eq. (A1) reads

$$
S_{2}\left(t_{b}\right)=-\frac{1}{2} \int_{t_{b}}^{t_{r}}\left[\mathbf{k}^{*}+\mathbf{A}(\tau)\right]^{2} d \tau+\left|E_{g^{+}}\right|\left(t_{r}-t_{b}\right)+\left|E_{g g}\right|\left(t_{b}-t_{i}\right) .
$$

Hence, the saddle point $t_{b}\left(t_{r}\right)$ is a function of $t_{r}$ and given as a solution of the following equation

$$
\frac{\cos \left(\omega t_{r}\right)-\cos \left[\omega t_{b}\left(t_{r}\right)\right]}{\omega\left[t_{r}-t_{b}\left(t_{r}\right)\right]}+\sin \left[\omega t_{b}\left(t_{r}\right)\right]= \pm i \gamma,
$$

where $\gamma$ is the Keldysh parameter for the first electron,

$$
\gamma=\frac{\omega \sqrt{2\left(E_{g^{+}}-E_{g g}\right)}}{F} .
$$


Methods of computing the saddle points [Eqs. (A3) and (A7)] have been widely discussed in the literature (see, for example, Refs. [31-33] and references therein). In the current paper, we use a general and simple approach for identifying correct saddle points between different solutions of the saddle-point equations in the complex plane (see Sec. IV).

It is convenient to introduce the following phases: $\phi_{b}=\omega t_{b}$ and $\varphi_{r}=\omega t_{r}$. According to Eq. (A3), the saddle point $\phi_{b}$ is a complex double-valued function of $\varphi_{r}$ which can be given by $\phi_{b}\left(+\gamma ; \varphi_{r}\right)$ and $\phi_{b}\left(-\gamma ; \varphi_{r}\right)$, where $\gamma$ is the Keldysh parameter (A4). Here, the complex singlevalued function $\phi_{b}\left(\gamma ; \varphi_{r}\right)$ is defined as a solution of the following transcendental equation:

$$
\frac{\cos \varphi_{r}-\cos \phi_{b}\left(\gamma ; \varphi_{r}\right)}{\varphi_{r}-\phi_{b}\left(\gamma ; \varphi_{r}\right)}+\sin \phi_{b}\left(\gamma ; \varphi_{r}\right)=i \gamma .
$$

No analytical solution of such an equation is available. The special case of the function $\phi_{b}\left(\gamma ; \varphi_{r}\right)$ for $\gamma=0$,

$$
\frac{\cos \varphi_{r}-\cos \varphi_{b}\left(\varphi_{r}\right)}{\varphi_{r}-\varphi_{b}\left(\varphi_{r}\right)}+\sin \varphi_{b}\left(\varphi_{r}\right)=0
$$

is very important because of the following two reasons.

First, the function $\varphi_{b}\left(\varphi_{r}\right)$ is a real valued function for real $\varphi_{r}$ (and single-valued for any complex $\varphi_{r}$ ); this allows one to interpret the motion of the first electron in terms of classical trajectories. The function $\varphi_{b}\left(\varphi_{r}\right)$ is defined on the interval $(\pi / 2,2 \pi]$ because only during that interval can the free electron recollide with its parent ion. The function $\varphi_{b}\left(\varphi_{r}\right)$ is bounded in the interval $0 \leqslant \varphi_{b}\left(\varphi_{r}\right)<\pi / 2$. Second, the function $\varphi_{b}\left(\varphi_{r}\right)$ can be physically understood as a tunneling limit $(\gamma \ll 1)$ of $\phi_{b}\left(\gamma ; \varphi_{r}\right)$ (i.e., the low-frequency limit).

In terms of the laser phase, the vector potential $\mathbf{A}(\varphi)$ is

$$
\mathbf{A}(\varphi)=-(\mathbf{F} / \omega) \sin \varphi
$$

The difference between Eq. (A5) and Eq. (A6), which connect the phase of recollision $\varphi_{r}$ with the phase of ionization $\varphi_{b}\left(\varphi_{r}\right)$ or $\phi_{b}\left(\gamma ; \varphi_{r}\right)$, is also important for the last step of NSDI - the release of the two electrons following the recollision at $\varphi_{r}$.

Calculating the integral over $t_{r}$ in Eq. (A1) by the SPA, we need to obtain the transition point $\varphi_{r}^{0}$ for negligible $\gamma$. It is the solution of the saddle-point equation

$$
\Delta E\left(\varphi_{r}^{0}\right) \equiv \frac{1}{2}\left[\mathbf{k}_{1}+\mathbf{A}\left(\varphi_{r}^{0}\right)\right]^{2}+\frac{1}{2}\left[\mathbf{k}_{2}+\mathbf{A}\left(\varphi_{r}^{0}\right)\right]^{2}-\frac{1}{2}\left[\mathbf{A}\left(\varphi_{r}^{0}\right)-\mathbf{A}\left(\varphi_{b}\left(\varphi_{r}^{0}\right)\right)\right]^{2}+I_{p}^{(2)}=0,
$$

such that

$$
\pi / 2<\operatorname{Re} \varphi_{r}^{0} \leqslant 2 \pi
$$


where $I_{p}^{(2)}=\left|E_{g^{+}}\right|$is the ionization potential of the second electron. For $\gamma \neq 0$, the equation is

$$
\Delta E\left(\gamma ; \varphi_{r}^{0}\right) \equiv \frac{1}{2}\left[\mathbf{k}_{1}+\mathbf{A}\left(\varphi_{r}^{0}\right)\right]^{2}+\frac{1}{2}\left[\mathbf{k}_{2}+\mathbf{A}\left(\varphi_{r}^{0}\right)\right]^{2}-\frac{1}{2}\left[\mathbf{A}\left(\varphi_{r}^{0}\right)-\mathbf{A}\left(\phi_{b}\left(\gamma ; \varphi_{r}^{0}\right)\right)\right]^{2}+I_{p}^{(2)}=0,
$$

where $\phi_{b}\left(\gamma ; \varphi_{r}^{0}\right)$ now depends on $\gamma$. Note that Eqs. (A7) and (A8) are basically Eq. (2) written in slightly different notations.

If the solution of Eq. (A7) on the interval $(\pi / 2,2 \pi]$ is real, then direct collisional ionization is possible. However, we are interested in the deep quantum regime when the following inequality is valid for the second electron:

$$
I_{p}^{(2)}>3.17 U_{p}
$$

By introducing the Keldysh parameter for the second electron $\gamma_{2}=\sqrt{\frac{I_{p}^{(2)}}{2 U_{p}}}$, we can write the last inequality as

$$
\gamma_{2}>1.26
$$

Equation (A9) physically means that the returning electron does not have enough energy to free the second electron.

When recollision energy is not sufficient for collisional ionization, transition requires help from the laser field. Mathematically, the arising integral is similar to those in the LandauDykhne (LD) theory (see Sec. II). The energy gap $\Delta E\left(\varphi_{r}\right)$ in Eqs. (A7) and (A8) plays the role of the transition energy for the LD transition [the term $E_{i}(t)-E_{f}(t)$ in Eq. (1)]. The peculiarity of $\Delta E\left(\gamma ; \varphi_{r}\right)$ given by Eq. (A8) is that it need not be real even for real $\varphi_{r}$, since in the term $\left[A\left(\varphi_{r}\right)-A\left(\phi_{b}\left(\gamma ; \varphi_{r}\right)\right)\right]^{2}$ the phase $\phi_{b}$ is complex. This subtle aspect underscores the important difference between using the solutions of Eq. (A5) or Eq. (A6) for the phase of ionization $\phi_{b}$. For classical trajectories, where $\varphi_{b}\left(\varphi_{r}\right)[\mathrm{Eq} .(\mathrm{A} 6)]$ is real for real $\varphi_{r}, \Delta E\left(\varphi_{r}\right)$ [Eq. (A7)] is also real for real $\varphi_{r}$. This is the standard assumption in the LD theory. When the complex phase of ionization $\phi_{b}\left(\gamma ; \varphi_{r}\right)[$ Eq. (A5)] is used, i.e., when "quantum" trajectories for recollision are used, $\Delta E\left(\gamma ; \varphi_{r}\right)[$ Eq. (A8)] need not be real for real $\varphi_{r}$. In [45], the LD method has been generalized for this case, provided that the complex function $\Delta E(\gamma ; \varphi)$ satisfies the Schwarz reflection principle (recently, this result was confirmed and further generalized in Ref. [46]).

The function $\Delta E(\varphi)$ (A7) obeys the Schwarz reflection principle, i.e., $\Delta E^{*}\left(\varphi^{*}\right)=\Delta E(\varphi)$. Hence, we can conclude that if $\varphi_{r}^{0}$ is the solution that lies in the lower half-plane, then $\left(\varphi_{r}^{0}\right)^{*}$ 
is the solution that lies in the upper half-plane. Furthermore, it can be easily proven that the following equation takes place for any function $\Delta E(\varphi)$ which satisfies the Schwarz reflection principle and any complex number $\varphi_{r}^{0}$ :

$$
\operatorname{Im} \int_{\operatorname{Re} \varphi_{r}^{0}}^{\left(\varphi_{r}^{0}\right)^{*}} \Delta E(\varphi) d \varphi=-\operatorname{Im} \int_{\operatorname{Re} \varphi_{r}^{0}}^{\varphi_{r}^{0}} \Delta E(\varphi) d \varphi .
$$

From the previous equation, we can see that the transition points that lie in the lower half-plane lead to exponentially large probabilities, which are unphysical. Hereafter, let $\varphi_{r}^{0}$ denote the solution of the equation $\Delta E\left(\varphi_{r}^{0}\right)=0$, which is the closest to the real axis and lies in the upper-half plane.

Before continuing our discussion, let us point out the following simple equalities, which follow from Eq. (A5): $\operatorname{Re}\left[\phi_{b}\left(+\gamma ; \varphi_{r}\right)\right]=\operatorname{Re}\left[\phi_{b}\left(-\gamma ; \varphi_{r}\right)\right]$ and $\operatorname{Im}\left[\phi_{b}\left(+\gamma ; \varphi_{r}\right)\right]=$ $-\operatorname{Im}\left[\phi_{b}\left(-\gamma ; \varphi_{r}\right)\right]$ for real $\varphi_{r}$. Furthermore, we obtain

$$
\phi_{b}^{*}\left(-\gamma ; \varphi_{r}^{*}\right)=\phi_{b}\left(\gamma ; \varphi_{r}\right), \quad \operatorname{Im} \int_{\operatorname{Re} \varphi_{r}^{0}}^{\left(\varphi_{r}^{0}\right)^{*}} \Delta E(\gamma ; \varphi) d \varphi=-\operatorname{Im} \int_{\operatorname{Re} \varphi_{r}^{0}}^{\varphi_{r}^{0}} \Delta E(-\gamma ; \varphi) d \varphi,
$$

where $E(\gamma ; \varphi)$ is defined in Eq. (A8).

Bearing in mind that formula (A1) must give an exponentially small result (which implies that the transition point must be located in the upper-half plane) and taking into account Eq. (A10), we define the phase of ionization in the case of $\gamma \neq 0$ as

$$
\Phi\left(\gamma ; \varphi_{r}\right)=\left\{\begin{array}{lll}
\phi_{b}\left(-\gamma ; \varphi_{r}\right) & \text { if } & \operatorname{Im}\left(\varphi_{r}\right)>0, \\
\operatorname{Re}\left[\phi_{b}\left(\gamma ; \varphi_{r}\right)\right] & \text { if } & \operatorname{Im}\left(\varphi_{r}\right)=0, \\
\phi_{b}\left(+\gamma ; \varphi_{r}\right) & \text { if } & \operatorname{Im}\left(\varphi_{r}\right)<0 .
\end{array}\right.
$$

Equation (A11) is the most consistent definition of the quantum-mechanical phase of ionization of the first electron as a function of the phase of return. Generally speaking, there was an ambiguity in selecting the value of $\Phi\left(\gamma ; \varphi_{r}\right)$ for real $\varphi_{r}$. However, we have chosen it in such a way due to the following reason. The function $\operatorname{Im}\left[\Phi\left(\gamma ; \varphi_{r}\right)\right]$ has a jump discontinuity on the real axis, but the function $\operatorname{Re}\left[\Phi\left(\gamma ; \varphi_{r}\right)\right]$ has a removable discontinuity that can be removed by employing the equality

$$
\operatorname{Re}\left[\phi_{b}\left(\gamma ; \varphi_{r}\right)\right] \equiv \frac{1}{2}\left[\phi_{b}\left(\gamma ; \varphi_{r}+i 0\right)+\phi_{b}\left(-\gamma ; \varphi_{r}-i 0\right)\right] \quad\left(\text { for real } \varphi_{r}\right) .
$$

Furthermore, the function $\Phi\left(\gamma ; \varphi_{r}\right)$ obeys the Schwarz reflection principle $\left[\Phi^{*}\left(\gamma ; \varphi_{r}^{*}\right)=\right.$ $\left.\Phi\left(\gamma ; \varphi_{r}\right)\right]$, and the following equality takes place:

$$
\Phi\left(0 ; \varphi_{r}\right)=\varphi_{b}\left(\varphi_{r}\right) .
$$


It is essential that according to definition (A11), the function $\Phi\left(\gamma ; \varphi_{r}\right)$ is real-valued on the real axis and thus allows the identical interpretation in terms of the classical trajectories as for $\varphi_{b}\left(\varphi_{r}\right)$. Therefore, the definition of $\varphi_{r}^{0}$ and inequality (A9) are unchanged in the case of $\gamma \neq 0$.

[1] P. B. Corkum, Phys. Rev. Lett. 71, 1994 (1993).

[2] M. Kuchiev, JETP Letters 45, 404 (1987).

[3] V. V. Suran and I. P. Zapesochny, Sov. Tech. Phys. Lett. 1, 420 (1975).

[4] I. I. Bondar' and V. V. Suran, JETP 76, 381 (1993).

[5] I. I. Bondar' and V. V. Suran, JETP Letters 68, 837 (1998).

[6] I. I. Bondar', V. V. Suran, and M. I. Dudich, J. Phys. B 33, 4243 (2000).

[7] P. Lambropoulos, X. Tang, P. Agostini, G. Petite, and A. L'Huillier, Phys. Rev. A 38, 6165 (1988).

[8] A. L'Huillier, L. A. Lompre, G. Mainfray, and C. Manus, Phys. Rev. Lett. 48, 1814 (1982).

[9] A. l'Huillier, L. A. Lompre, G. Mainfray, and C. Manus, Phys. Rev. A 27, 2503 (1983).

[10] B. Walker, E. Mevel, B. Yang, P. Breger, J. P. Chambaret, A. Antonetti, L. F. DiMauro, and P. Agostini, Phys. Rev. A 48, R894 (1993).

[11] B. Walker, B. Sheehy, L. F. DiMauro, P. Agostini, K. J. Schafer, and K. C. Kulander, Phys. Rev. Lett. 73, 1227 (1994).

[12] A. Rudenko, K. Zrost, B. Feuerstein, V. L. B. de Jesus, C. D. Schröter, R. Moshammer, and J. Ullrich, Phys. Rev. Lett. 93, 253001 (2004).

[13] K. Zrost, A. Rudenko, T. Ergler, B. Feuerstein, V. L. B. de Jesus, C. D. Schröter, R. Moshammer, and J. Ullrich, J. Phys. B 39, S371 (2006).

[14] K. T. Taylor, J. S. Parker, D. Dundas, and K. J. Meharg, J. Mod. Opt. 54, 1959 (2007).

[15] A. Staudte, C. Ruiz, M. Schöffler, S. Schössler, D. Zeidler, T. Weber, M. Meckel, D. M. Villeneuve, P. B. Corkum, A. Becker, et al., Phys. Rev. Lett. 99, 263002 (2007).

[16] A. Rudenko, V. L. B. de Jesus, T. Ergler, K. Zrost, B. Feuerstein, C. D. Schröter, R. Moshammer, and J. Ullrich, Phys. Rev. Lett. 99, 263003 (2007).

[17] Y. Liu, S. Tschuch, A. Rudenko, M. Dürr, M. Siegel, U. Morgner, R. Moshammer, and J. Ullrich, Phys. Rev. Lett. 101, 053001 (2008). 
[18] D. Zeidler, A. Staudte, A. B. Bardon, D. M. Villeneuve, R. Dorner, and P. B. Corkum, Phys. Rev. Lett. 95, 203003 (2005).

[19] M. Weckenbrock, D. Zeidler, A. Staudte, T. Weber, M. Schöffler, M. Meckel, S. Kammer, M. Smolarski, O. Jagutzki, V. R. Bhardwaj, et al., Phys. Rev. Lett. 92, 213002 (2004).

[20] R. Panfili, S. L. Haan, and J. H. Eberly, Phys. Rev. Lett. 89, 113001 (2002).

[21] P. J. Ho and J. H. Eberly, Phys. Rev. Lett. 95, 193002 (2005).

[22] P. J. Ho, R. Panfili, S. L. Haan, and J. H. Eberly, Phys. Rev. Lett. 94, 093002 (2005).

[23] S. L. Haan, L. Breen, A. Karim, and J. H. Eberly, Phys. Rev. Lett. 97, 103008 (2006).

[24] S. L. Haan, V. J. S. Dyke, and Z. S. Smith, Phys. Rev. Lett. 101, 113001 (2008).

[25] P. J. Ho and J. H. Eberly, Phys. Rev. Lett. 97, 083001 (2006).

[26] A. Becker and F. H. M. Faisal, J. Phys. B 38, R1 (2005).

[27] G. L. Yudin and M. Y. Ivanov, Phys. Rev. A 63, 033404 (2001).

[28] M. Lein, E. K. U. Gross, and V. Engel, Phys. Rev. Lett. 85, 4707 (2000).

[29] C. F. de Morisson Faria, arXiv:0807.2763v1 [physics.atom-ph].

[30] C. F. de Morisson Faria, T. Shaaran, X. Liu, and W. Yang, arXiv:0806.4856v2 [physics.atomph].

[31] F. C. de Morisson Faria and M. Lewenstein, J. Phys. B 38, 3251 (2005).

[32] F. C. de Morisson Faria, X. Liu, A. Sanpera, and M. Lewenstein, Phys. Rev. A 70, 043406 (2004).

[33] F. C. de Morisson Faria, X. Liu, W. Becker, and H. Schomerus, Phys. Rev. A 69, 021402 (2004).

[34] F. C. de Morisson Faria and W. Becker, Laser Physics 13, 1196 (2003).

[35] X. Liu, F. C. de Morisson Faria, W. Becker, and P. B. Corkum, J. Phys. B 39, L305 (2006).

[36] F. C. de Morisson Faria, X. Liu, and W. Becker, J. Mod. Opt. 53, 193 (2006).

[37] O. Smirnova, M. Spanner, and M. Ivanov, Phys. Rev. A 77, 033407 (2008).

[38] V. R. Bhardwaj, S. A. Aseyev, M. Mehendale, G. L. Yudin, D. M. Villeneuve, D. M. Rayner, M. Y. Ivanov, and P. B. Corkum, Phys. Rev. Lett. 86, 3522 (2001).

[39] A. M. Dykhne, Sov. Phys. JETP 14, 941 (1962).

[40] L. D. Landau and E. M. Lifshitz, Quantum mechanics: non-relativistic theory (Oxford; Toronto: Pergamon, 1977).

[41] N. B. Delone and V. P. Krainov, Atoms in strong light fields (Berlin: Springer-Verlag, 1985). 
[42] A. V. Chaplik, Sov. Phys. JETP 18, 1046 (1964).

[43] J. P. Davis and P. Pechukas, J. Chem. Phys. 64, 3129 (1976).

[44] A. Joye and C.-E. Pfister, Phys. Rev. A 48, 2598 (1993).

[45] C. A. Moyer, Phys. Rev. A 64, 033406 (2001).

[46] R. Schilling, M. Vogelsberger, and D. A. Garanin, J. Phys. A 39, 13727 (2006).

[47] D. I. Bondar, Phys. Rev. A 78, 015405 (2008).

[48] V. S. Rastunkov and V. P. Krainov, J. Phys. B 40, 2277 (2007).

[49] V. P. Krainov, J. Phys. B 36, L169 (2003).

[50] G. L. Yudin and M. Y. Ivanov, Phys. Rev. A 64, 013409 (2001).

[51] N. B. Delone and V. P. Krainov, J. Opt. Soc. Am. B 8, 1207 (1991).

[52] A. M. Perelomov, V. S. Popov, and M. V. Terent'ev, Sov. Phys. JETP 23, 924 (1966).

[53] A. M. Perelomov, V. S. Popov, and M. V. Terent'ev, Sov. Phys. JETP 24, 207 (1967).

[54] A. M. Perelomov and V. S. Popov, Sov. Phys. JETP 25, 336 (1967).

[55] V. S. Popov, V. P. Kuzentsov, and A. M. Perelomov, Sov. Phys. JETP 26, 222 (1968).

[56] S. V. Popruzhenko, G. G. Paulus, and D. Bauer, Phys. Rev. A 77, 053409 (2008).

[57] S. V. Popruzhenko and D. Bauer, J. Mod. Opt. 55, 2573 (2008).

[58] O. Smirnova, A. S. Mouritzen, S. Patchkovskii, and M. Y. Ivanov, J. Phys. B 40, F197 (2007).

[59] V. S. Popov, Physics of Atomic Nuclei 68, 686 (2005).

[60] V. S. Popov, Physics-Uspekhi 47, 855 (2004).

[61] B. Feuerstein, R. Moshammer, D. Fischer, A. Dorn, C. D. Schröter, J. Deipenwisch, J. R. Crespo Lopez-Urrutia, C. Höhr, P. Neumayer, J. Ullrich, et al., Phys. Rev. Lett. 87, 043003 (2001).

[62] V. L. B. de Jesus, B. Feuerstein, K. Zrost, D. Fischer, A. Rudenko, F. Afaneh, C. D. Schröter, R. Moshammer, and J. Ullrich, J. Phys. B 37, L161 (2004). 

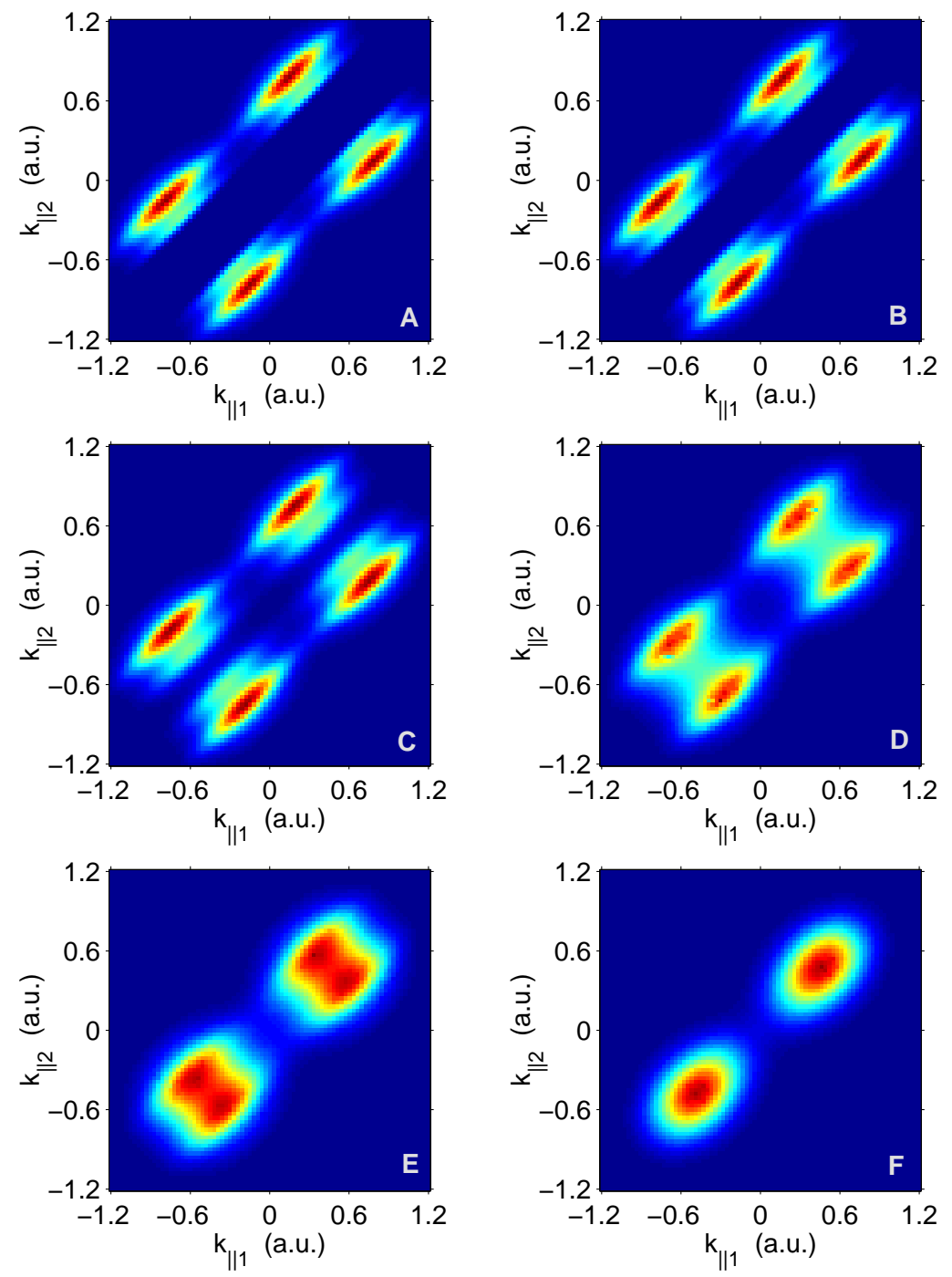

FIG. 3: Role of electron-electron interaction. Correlated spectra of Ar (linear scale) at $7 \times 10^{13}$ $\mathrm{W} / \mathrm{cm}^{2}, 800 \mathrm{~nm}$ are calculated using Eq. (17) with $r_{12}^{(0)}=0.985$ a.u. $(\gamma=1.373)$. Electron-core interaction is not included. Spectra are shown for different values of $k_{\perp}$ (in a.u.) for both electrons: (a) $k_{\perp 1}=k_{\perp 2}=0$; (b) $k_{\perp 1}=0, k_{\perp 2}=0.2$; (c) $k_{\perp 1}=-0.1, k_{\perp 2}=0.2$; (d) $k_{\perp 1}=-0.2, k_{\perp 2}=0.3$; (e) $k_{\perp 1}=-0.3, k_{\perp 2}=0.3$; (f) $k_{\perp 1}=-0.5, k_{\perp 2}=0.5$. Maxima of figures correspond to probability densities: (a) $9.1 \times 10^{-19}$, (b) $6.5 \times 10^{-19}$, (c) $8.36 \times 10^{-19}$, (d) $8.4 \times 10^{-19}$, (e) $9.1 \times 10^{-19}$, (f) $2.8 \times 10^{-20}$. 

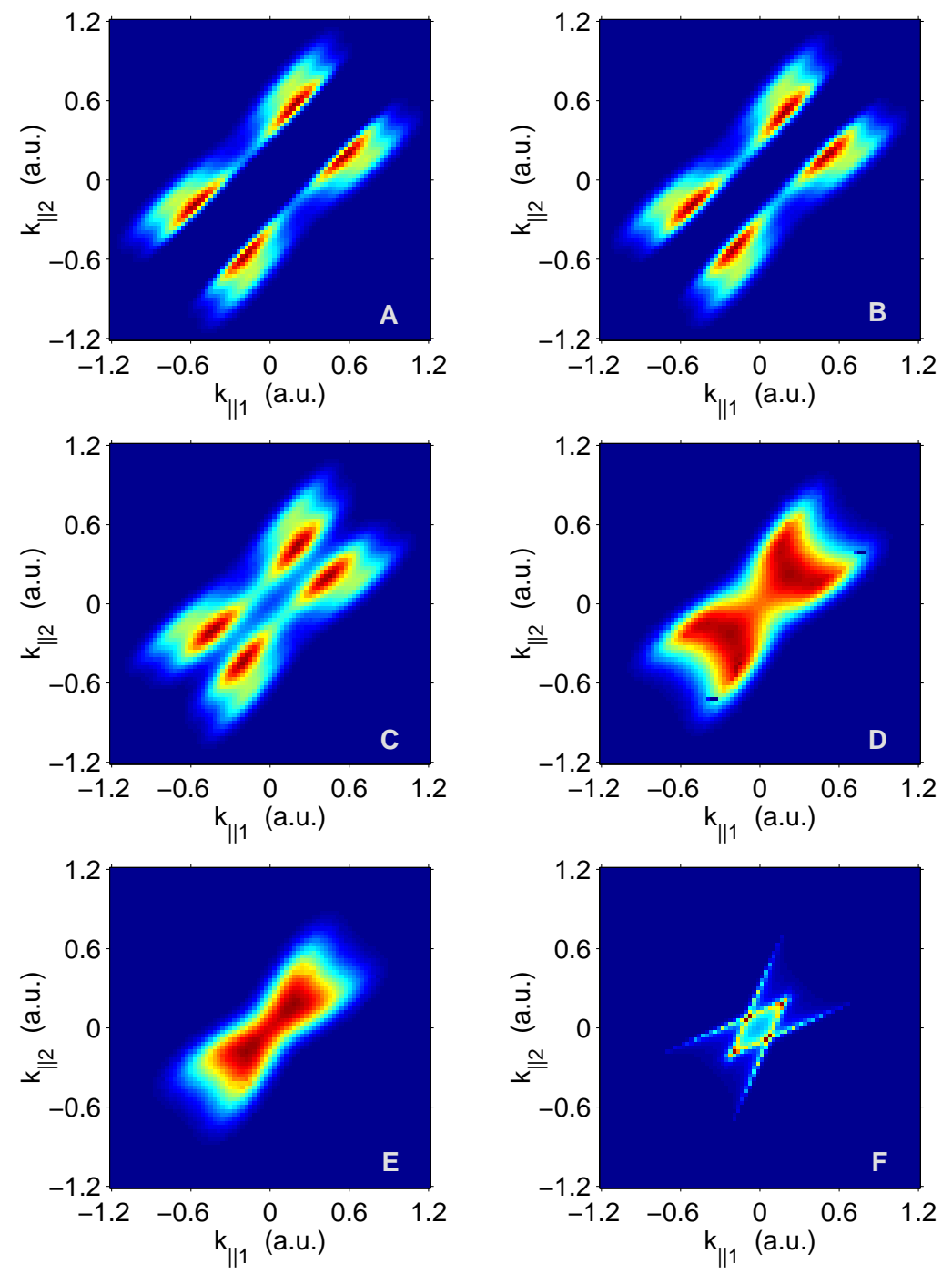

FIG. 4: Role of electron-core interaction. Correlated spectra of $\mathrm{Ar}$ (linear scale) at $7 \times 10^{13} \mathrm{~W} / \mathrm{cm}^{2}$, $800 \mathrm{~nm}$ are calculated using Eq. (19) with $r_{12}^{(0)}=r_{0}=0.985$ a.u. $(\gamma=1.373)$. Both electronelecron and electron-core interactions are included. Spectra are shown for different values of $k_{\perp}$ (in a.u.) for both electrons: (a) $k_{\perp 1}=k_{\perp_{2}}=0$; (b) $k_{\perp 1}=0, k_{\perp 2}=0.2$; (c) $k_{\perp 1}=-0.1, k_{\perp 2}=0.2$; (d) $k_{\perp 1}=-0.2, k_{\perp 2}=0.3$; (e) $k_{\perp 1}=-0.3, k_{\perp 2}=0.3$; (f) $k_{\perp 1}=-0.5, k_{\perp 2}=0.5$. Maxima of figures correspond to probability densities: (a) $2.8 \times 10^{-5}$, (b) $3.3 \times 10^{-5}$, (c) $6.1 \times 10^{-5}$, (d) $1.5 \times 10^{-4}$, (e) $4.4 \times 10^{-4}$, (f) $7.7 \times 10^{-4}$. 